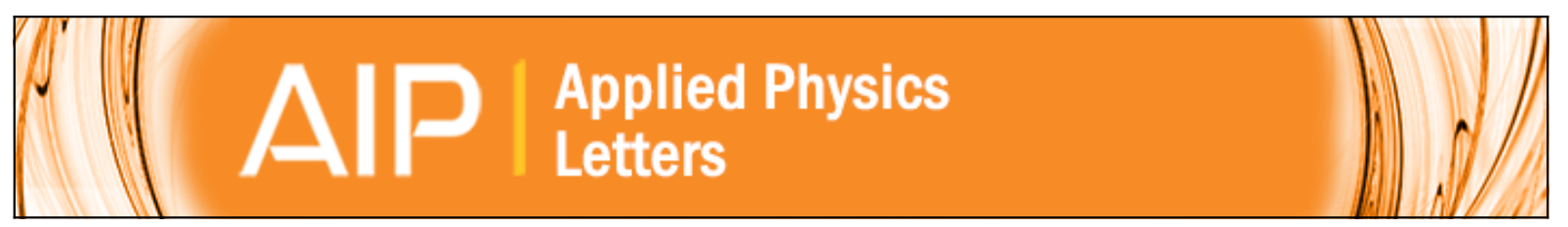

\title{
Noise characterization of metal-single molecule contacts
}

D. Xiang, V. Sydoruk, S. Vitusevich, M. V. Petrychuk, A. Offenhäusser, V. A. Kochelap, A. E. Belyaev, and D. Mayer

Citation: Applied Physics Letters 106, 063702 (2015); doi: 10.1063/1.4908252

View online: http://dx.doi.org/10.1063/1.4908252

View Table of Contents: http://scitation.aip.org/content/aip/journal/apl/106/6?ver=pdfcov

Published by the AIP Publishing

\section{Articles you may be interested in}

Measurement and understanding of single-molecule break junction rectification caused by asymmetric contacts J. Chem. Phys. 141, 054712 (2014); 10.1063/1.4891862

Noise and transport characterization of single molecular break junctions with individual molecule J. Appl. Phys. 112, 014908 (2012); 10.1063/1.4736558

Fabrication and characterization of metal-molecule-silicon devices Appl. Phys. Lett. 91, 033508 (2007); 10.1063/1.2750516

Nanowell device for the electrical characterization of metal-molecule-metal junctions J. Vac. Sci. Technol. B 23, 1417 (2005); 10.1116/1.1935528

Nature of electrical contacts in a metal-molecule-semiconductor system J. Vac. Sci. Technol. B 21, 1928 (2003); 10.1116/1.1588641

\section{Model PS-100}

Tabletop Cryogenic Probe Station

\section{Lake Shore CRYOTRONICS}

An affordable solution for a wide range of research 


\title{
Noise characterization of metal-single molecule contacts
}

\author{
D. Xiang, ${ }^{1,4, a)}$ V. Sydoruk, ${ }^{1, a)}$ S. Vitusevich, ${ }^{1, b)}$ M. V. Petrychuk, ${ }^{2}$ A. Offenhäusser, ${ }^{1}$ \\ V. A. Kochelap, ${ }^{3}$ A. E. Belyaev, ${ }^{3}$ and D. Mayer $\left.{ }^{1, b}\right)$ \\ ${ }^{1}$ Peter-Grünberg-Institute (PGI-8), Forschungszentrum Jülich, 52425 Jülich, Germany \\ ${ }^{2}$ Taras Shevchenko National University of Kyiv, 03022 Kiev, Ukraine \\ ${ }^{3}$ Institute of Semiconductor Physics, NASU, 03028 Kiev, Ukraine \\ ${ }^{4}$ Key Laboratory of Optical Information Science and Technology, Institute of Modern Optics, \\ Nankai University, Ministry of Education, Tianjin 300071, China
}

(Received 23 December 2014; accepted 4 February 2015; published online 12 February 2015)

\begin{abstract}
Noise spectra of molecule-free and molecule-containing mechanically controllable break junctions were investigated. The molecule-free junctions revealed typical $1 / f$ noise characteristics. We studied three molecules as they bridged the electrodes: 11-mercaptoundecanoicacid, 1,8-octanedithiol, and 1,4-benzenedithiol, which possess different bonding strengths. For all of them, an additional Lorentzian-shape $1 / f^{2}$ noise component was registered with a characteristic frequency when the electrodes were bridged by an individual molecule. Measurements of time-dependent voltage fluctuations for the molecule-containing junctions bring out two-current state fluctuations, which in the frequency domain correspond to the $1 / f^{2}$ noise. Moreover, it is revealed that characteristic frequencies of these noise components are independent of molecule bonding strengths at the interface, but correlate with the molecule weights and current amplitudes in the lock-in state, in which the electrode gap is bridged by a single molecule. We attribute the noise monitored during charge transport through a molecular junction to the current induced molecular reconfigurations and suggest that the noise analysis can be used for characterization of metal-molecule coupling. (C) 2015 AIP Publishing LLC. [http://dx.doi.org/10.1063/1.4908252]
\end{abstract}

Driven by the interest in fundamental aspects of the processes in molecular electronic devices, theoretical and experimental investigations of charge transport through molecules attract increasing attention. During the last decade, experimental and theoretical advances have yielded significant insight into electron transport in metal-molecule-metal junctions. ${ }^{1-4}$ In particular, individual molecule charge transport investigations became feasible by realization of different nanoelectrode configurations, where the electrodes are separated by a gap of molecular dimensions. ${ }^{5-11}$ Breakjunction setups with tunable distance between the nanocontacts such as scanning tunneling microscopy, atomic force microscopy, and mechanically controllable break junctions (MCBJs) provide control over the number of molecules connected in the junction. The recent MCBJ achievements are reviewed in Ref. 12.

Recently, metal-molecule junctions have attracted particular attention (see, for example, Refs. 12 and 13 and references therein) because of importance achieving reliable contacts for molecular electronics. Noise spectroscopy is a method of studying the performance of materials and devices, including also metal-molecule contacts, which is still a poorly explored area of research. Random telegraph-like current fluctuations with large amplitude variation $(>60 \%)$ were observed under relatively high-voltages (about $200 \mathrm{mV}$ ) in Ref. 13. These current fluctuations were attributed to thermally activated formation and breaking of bonds between a molecule and a metal electrode.

\footnotetext{
${ }^{a)}$ D.X. and V.S. contributed equally to this work.

b) Authors to whom correspondence should be addressed. Electronic addresses: s.vitusevich@fz-juelich.de and dirk.mayer@fz-juelich.de
}

The noise spectroscopy applied at small voltages (about $20 \mathrm{mV}$ ) can provide insight into fundamental problems concerning conductivity formation mechanism in metalmolecule contacts since small time-dependent changes in a contact between the molecule and the metal electrode can appear in the fluctuations of the current. In our previous report, ${ }^{14}$ a Lorentzian-shape noise component (in addition to the $1 / f$ noise and the thermal noise characteristic for the molecule-free device) was registered by studying highstability break junction devices with 1,4-Benzenediamine (BDA) molecules. This noise component, which corresponds to telegraph-like current fluctuations, was observed under relatively small bias voltages. We interpret the observed Lorentzian-type noise as the manifestation of a system reconfiguration under the transport of charge across a single molecule. Experimental results obtained in Ref. 14, particularly dependencies of the noise amplitude and the characteristic noise frequency on the current, support the explanation of the observed Lorentzian-type noise as a demonstration of a dynamic reconfiguration of molecular coupling to the metal electrodes.

In this communication, we present results of the study of several molecules with different coupling strengths and show that the observed Lorentzian-shape noise is of general phenomenon. We found that for different bonding groups, the amplitude of current fluctuations does not exceed $12 \%$. Moreover, we reveal that for different molecules, the characteristic frequency of the noise component is independent of molecule bonding strength at the interface, but correlates with the current amplitudes in the lock-in state, in which the electrode gap is bridged by a single molecule. In contrast to Ref. 13, our results indicate that telegraph fluctuations 
originate from the current induced reconfigurations of the molecule. In addition, we found that the molecule's characteristic frequencies correlate with their molecular weights.

The MCBJ-technique was used for achieving the high stability metal-molecule-metal junction. In order to reach high stability in the junction, a three point bending apparatus was developed in-house with a very fine tuning system. A thin spring steel substrate containing a suspended nano-sized metal constriction was mounted into the system, as shown in Fig. 1. The two outer posts of the bending apparatus were fixed while the third post worked as a pushing rod, driven in the vertical direction by piezoelectric actuator. When the push rod moved in the vertical direction, a bending force was exerted on the substrate, which caused an elongation of the constriction until the metal bridge broke, resulting in two separated nanoelectrodes. The distance between two electrodes can be very precisely tuned by bending or relaxing the substrate. Precision control of the gap size is determined by the attenuation factor, the gap size change between two electrodes divided by the displacement of the push rod. For the setup used, an attenuation factor of $r \sim 10^{-6}$ was achieved. After calibration of the molecule-free junction, 1,8-octanedithiol (ODT), 1,4-benzenedithiol (BDT), or 11-mercaptoundecanoic acid (MUA) were adsorbed and self-assembled on the gold wire from solution, dried with argon and subsequently mounted into the MCBJ setup. The metal-molecule-metal junction state can be found by bending the sample and simultaneously monitoring the conductance change of the system. ${ }^{12,14}$ When the molecular bridge junction was obtained, the noise characterization investigations were performed in vacuum at room temperature $(\mathrm{T}=298 \mathrm{~K})$.

We applied this high-stability MCBJ technique, and the noise measurement protocol described in Ref. 14 to study different types of molecules. ODT, BDT, 1,4-Benzenediamine (BDA), and 11-mercaptoundecanoicacid (MUA) with different bonding groups were selected in the following order of

\section{(a)}
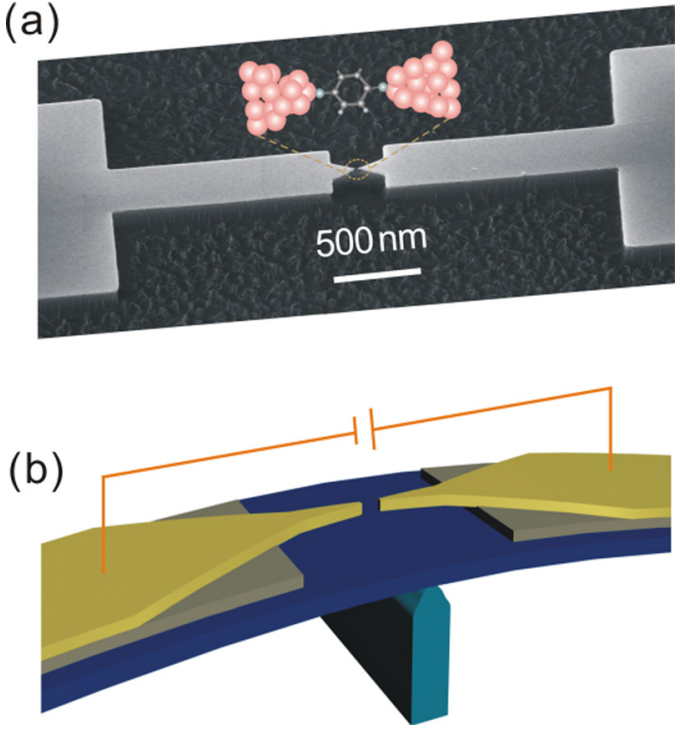

FIG. 1. Strategy to form stable single-molecule junction with MCBJ. (a) Scanning electron microscopy image of a nanofabricated MCBJ chip. Inset: Schematic drawing of two nano-electrodes bridged by a molecule. (b) Schematic drawing of MCBJ sample mounted on a three point bending apparatus. binding strength: $-\mathrm{S}>-\mathrm{NH}>-\mathrm{COOH}$. For each of the molecules, we measured at least 3 different MCBJs. The noise spectrum was obtained by averaging 100 curves using a spectrum analyzer. For the molecule-free gap junction, we observed only the $1 / f$ and the thermal components of the current noise. When a molecule bridged the electrodes, in addition to the two noise components mentioned, we observed $1 / \mathrm{f}^{2}$ noise. Thus, the spectral density of the overall noise can be described as

$$
S_{V}(f)=\frac{A}{f}+\frac{B}{1+\left(f / f_{0}\right)^{2}}+4 \mathrm{kTR} .
$$

Here, A and $B$ represent the amplitudes of $1 / f$ and $1 / f^{2}$ noise components, respectively, $f_{0}$ is the characteristic frequency derived from the inflection position of the total noise spectra, $R$ is the equivalent resistance (see details in Ref. 14), and $T$ is the ambient temperature. Equation (1) fits the experimental data well, as illustrated in Fig. 2 for the BDT molecule. For this case, the characteristic frequency $f_{0}$ was found to be $1.2 \mathrm{kHz}$.

In these studies, we found, that $1 / f^{2}$ noise components are general features in noise behavior for different types of molecules. Thus, despite different electronic structures, bond strengths, and bonding groups for all studied molecules, the $1 / f^{2}$ - noise component was found. The measured characteristic frequencies $\left(f_{0}\right)$ and molecular parameters are summarized in Table I. From this table, one can see that there is no correlation between the characteristic noise frequencies and the bonding strength, or the bonding type. These results indicate that the registered characteristic frequency and telegraph fluctuations (described in more detail below) unlikely originate from the break and reconnection of the moleculeelectrode contact in our experiment. Moreover, we found that $f_{0}$ increases as the energy parameter, $\left(E_{F}-E_{M O}\right)$, of the molecular junctions decreases. This is reasonable, since the decrease of $\left(E_{F}-E_{M O}\right)$ will lead to an increase in tunneling current, and the increase in tunneling current will result in faster fluctuations, and therefore in increased $f_{0}$.

We further performed measurements of time-dependent voltage fluctuations for the molecule-free and molecule-

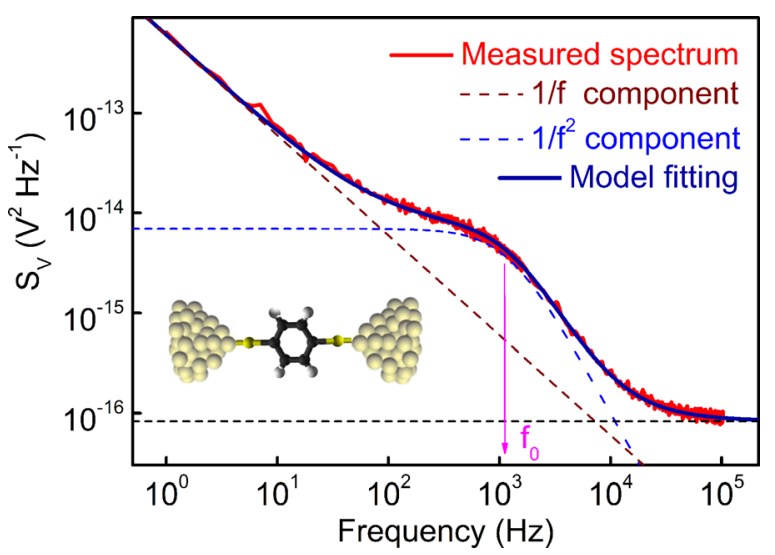

FIG. 2. The voltage noise power spectral density of the molecule containing junction in the lock-in state for a single BDT molecule bridging two electrodes (cartoon). The solid red curve is the measured noise density, and the solid blue curve represents the model according to Eq. (1). The $1 / f, 1 / f^{2}$, and thermal noise components are shown by dashed lines. 
TABLE I. Electronic structure, characteristic noise frequency, bond strength, and bonding groups for studied molecules. The current can also be estimated, according to the formula $\mathrm{I}=\mathrm{V} / \mathrm{R}$. Here, $\mathrm{V}=20 \mathrm{mV}$, and $\mathrm{R}$ is the resistance of each type of molecule.

\begin{tabular}{lcccc}
\hline \hline Molecules & $\begin{array}{c}E_{\mathrm{F}}-E_{\mathrm{HOMO}} \\
(\mathrm{eV})^{\mathrm{a}}\end{array}$ & $\begin{array}{c}f_{0} \\
(\mathrm{KHz})\end{array}$ & $\begin{array}{c}\text { Bond strength } \\
(\mathrm{KJ} / \mathrm{mol})^{\mathrm{b}}\end{array}$ & $\begin{array}{c}\text { Bond } \\
\text { type }\end{array}$ \\
\hline MUA & 1.8 & $0.07 \pm 0.01$ & 20 & $\mathrm{Au}-\mathrm{COOH}$ \\
ODT & 1.4 & $0.27 \pm 0.03$ & 134 & $\mathrm{Au}-\mathrm{S}$ \\
BDT $_{\text {BDA }^{\mathrm{c}}}^{1.2}$ & 1.0 & $1.1 \pm 0.2$ & 134 & $\mathrm{Au}-\mathrm{S}$ \\
\hline \hline
\end{tabular}

${ }^{\mathrm{a}}\left[E_{\mathrm{F}}-E_{\mathrm{HOMO}}\right]$ is the energy gap between the Fermi level of the gold electrodes and HOMO state of the molecules (taken from Refs. 17-19).

${ }^{\mathrm{b}}$ See Ref. 20.

${ }^{\mathrm{c}}$ From Ref. 14-given for comparison. For information on the resistance of each type of molecule-see Refs. 5, 14, 17, and 21.

containing junctions. The results are shown in Fig. 3 for the BDT molecule (the fixed voltage was $20 \mathrm{mV}$ ). No characteristic features were found for the molecule-free junctions. Instead, two states of the current via the single molecule, high-current and low current, were observed for the molecule-containing junctions. The high and low current states differ slightly by $\Delta I \approx 1.5 \mathrm{nA}$ at the average current $I \approx 14 \mathrm{nA}$. The small amplitudes of the current fluctuations (less than 12\%) further indicate that these two-level fluctuations cannot be attributed to a transition between the break and the reconnection of the molecule at the bonding interface, since the break of molecule junction should give rise to much larger amplitudes of the current fluctuations. ${ }^{6-8}$

It should be noted that large-amplitude fluctuations (over 60\%) were observed in liquid conditions in Ref. 13 at high voltage amplitudes (around $200 \mathrm{mV}$ ). The authors attributed these fluctuations to thermally driven breaking of bonds between a molecule and a metal electrode. In our case, the experiment was performed in the single molecule lock-in state at small applied biases and under vacuum conditions. There was no large-amplitude fluctuation observed in the molecular junctions with different bonding groups. The amplitude of typical fluctuations does not exceed $12 \%$ for all types of junctions. This additionally confirms that the underlying processes take place in the system without completely breaking the bond. The molecule-metal contact

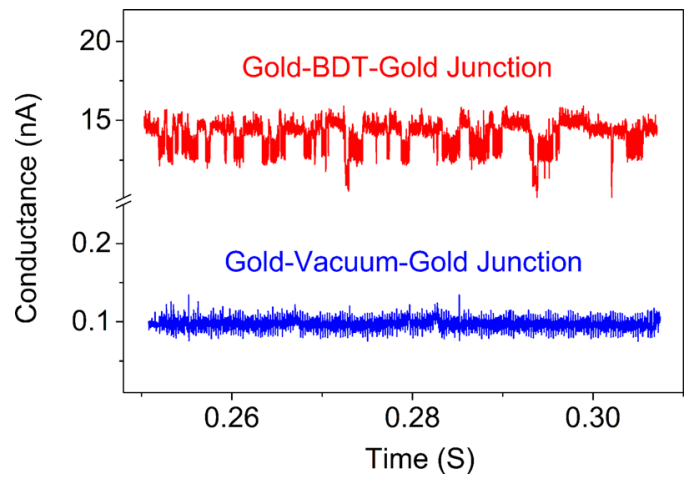

FIG. 3. Time dependence of the current measured at the applied voltage $\mathrm{V}_{\mathrm{B}}=20 \mathrm{mV}$. Two-level fluctuations are observed when a nanogap is bridged by single BDT molecule (red curve). Two level fluctuations are absent in the molecule-free junction (blue curve). The gap size between the electrodes was set to approximately $1 \mathrm{~nm}$ in both cases. involves near-configuration states separated by a low energy barrier and has very similar bonding coupling and electric properties, which agrees with the analysis given in Ref. 14 and 15 . In the frequency domain, these small two-state current fluctuations correspond to the voltage $1 / f^{2}$ noise component.

In the Landau theory, ${ }^{16}$ non-equilibrium processes (i.e., the current in our case) are responsible for long relaxation times of the order of milliseconds. Let us consider the processes in molecule-containing mechanically controlled break junctions. All existing times typical for the motion and relaxation of the electrons and nuclei in a molecule and metal contacts are smaller by many orders of magnitude than the characteristic times of the noise recorded. We assumed that the characteristic noise times are related to slow processes induced by low currents via the electrode-molecule system. Indeed, when a current flows through a molecule, its electron subsystem becomes polarized, which induces small structural/configuration changes. Thus, charge transfer and structural/configuration changes are coupled. Small currents generate only small forces inducing these changes, and therefore, large characteristic times of such reconfigurations. According to the Landau theory, ${ }^{16}$ at non-equilibrium conditions, a system with two sets of strongly distinct characteristic times can demonstrate specific relaxation and time-dependent fluctuations with the Lorentzian-type spectral density. Introducing a slow configuration coordinate responsible for molecule-electrode coupling, we developed a phenomenological model that takes into account a correlation between charge transfer via a single molecule and structural changes in the coupling. The larger the current, larger characteristic fluctuation frequencies (the faster relaxation) will be obtained. In Ref. 14, we found that for BDA, both the plateau of Lorentzian-shape noise component, $S_{V 0}^{L S}$, and the characteristic frequency, $f_{0}$, are linearly dependent on current.

Fig. 4 shows the measured $f_{O}$ as a function of the current for the BDT molecule. Note that the linear increase of $f_{0}$ with the current (the larger the current, the faster the fluctuations) is confirmed. Similar linear behavior was also found for other molecules, which reflects common characteristic features for the molecules studied. Furthermore, we found that the fluctuation ratio is inversely proportional to the molecule's weight. If $M$ denotes the Molar mass of a molecule,

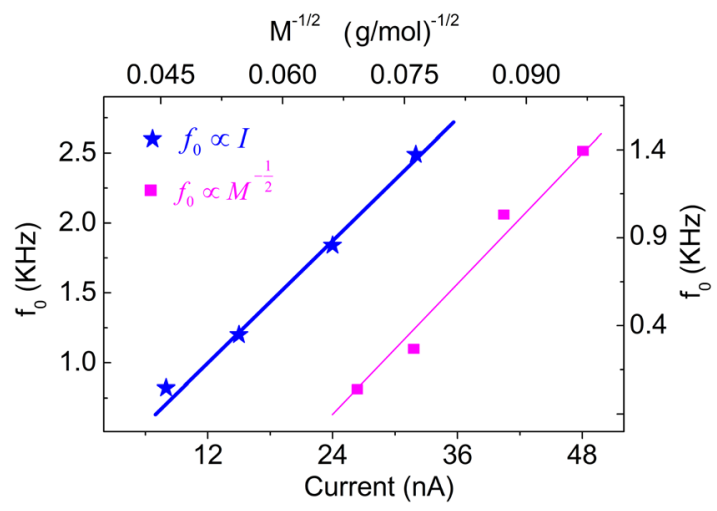

FIG. 4. Dependence of the characteristic frequency $f_{0}$ on the current $I$ for the BDT containing junction (blue stars); $f_{0}$ versus $1 / M^{1 / 2}$ for the molecules presented in Table I at the current $I=15 \mathrm{nA}$ (red square). 
we find that $f_{O} \sim M^{-1 / 2}$ at a given current value, as shown in Fig. 4. It agrees well with the fact that light molecules can more easily change configuration in the system. This finding further reflects that for each molecule type, structural changes in the coupling are responsible for the appearance of random telegraph current fluctuations in the single molecule junction. Understanding the conductance fluctuations and corresponding noise characteristics in the single molecule junction is of great significance for realizing molecular-scale logic circuits, especially for molecular switches and memories. Our systematic studies allow discovery of the underlying mechanism of the conductance fluctuations in single-molecule junctions, and unambiguously demonstrate that conductance fluctuations mainly originate from structural changes in the molecular coupling.

In summary, we measured and compared the electronic noise of molecule-free and single-molecule-containing mechanically controllable break junctions for several molecules with different bonding strengths. Both junctions revealed typical $1 / f$ noise characteristics. An additional $1 / f^{2}$ noise component was clearly observed only in metal $/ \mathrm{mole}$ cule/metal junctions when a single molecule bridged the nanoelectrodes. We found that in the time domain, this noise component corresponds to small two-state current fluctuations. The comparison of the noise spectra obtained for different molecules revealed that the characteristic frequency of these $1 / f^{2}$ noise components is independent of the molecule's bonding strength at the interface. At the same time, the frequency correlates with the lock-in current amplitude and molecular weight. This confirms that the $1 / f^{2}$ electric noise component relevant to a single bridging molecule corresponds to current induced dynamic reconfigurations of molecular coupling to the metal electrodes. The observed noise features can be described by the general Landau model that phenomenologically takes into account a correlation between charge transfer via a single molecule and structural changes in the coupling. We suggest that noise analysis can be used for characterization of coupling processes between metal and molecule.
Financial support from National Natural Science Foundation of China (No. 21303171) is gratefully acknowledged. This work was supported by the Federal Ministry of Education and Research (BMBF Project 01DK13016). We thank V. Maybeck for proofreading and comments on the manuscript.

${ }^{1}$ J. E. Green, J. W. Choi, A. Boukai, Y. Bunimovich, E. Johnston-Halperin, E. DeIonno, Y. Luo, B. A. Sheriff, K. Xu, Y. S. Shin, H. R. Tseng, J. F. Stoddart, and J. R. Heath, Nature 445, 414 (2007).

${ }^{2}$ M. Galperin, M. A. Ratner, A. Nitzan, and A. Troisi, Science 319, 1056 (2008).

${ }^{3}$ J. G. Kushmerick, J. Naciri, J. C. Yang, and R. Shashidhar, Nano Lett. 3, 897 (2003).

${ }^{4}$ X. D. Chen, S. Yeganeh, L. D. Qin, S. Z. Li, C. Xue, A. B. Braunschweig, G. C. Schatz, M. A. Ratner, and C. A. Mirkin, Nano Lett. 9, 3974 (2009).

${ }^{5}$ D. Xiang, H. Jeong, D. Kim, T. Lee, Y. Cheng, Q. Wang, and D. Mayer, Nano Lett. 13, 2809 (2013).

${ }^{6}$ E. Lörtscher, H. B. Weber, and H. Riel, Phys. Rev. Lett. 98, 176807 (2007).

${ }^{7}$ S. Wu, M. T. González, R. Huber, S. Grunder, M. Mayor, C. Schönenberger, and M. Calame, Nat. Nanotechnol. 3, 569 (2008).

${ }^{8}$ C. A. Martin, D. Ding, H. S. J. van der Zant, and J. M. van Ruitenbeek, New J. Phys. 10, 065008 (2008).

${ }^{9}$ T. A. Jung, R. R. Schlittler, and J. K. Gimzewski, Nature 386, 696 (1997).

${ }^{10}$ L. Grüter, M. T. González, R. Huber, M. Calame, and C. Schönenberger, Small 1, 1067 (2005).

${ }^{11}$ Z. F. Huang, F. Chen, R. D'Agosta, P. A. Bennett, M. Di. Ventra, and N. J. Tao, Nat. Nanotechnol. 2, 698 (2007).

${ }^{12}$ D. Xiang, H. Jeong, T. Lee, and D. Mayer, Adv. Mater. 25, 4845 (2013).

${ }^{13}$ J. Brunner, M. T. González, C. Schönenberger, and M. Calame, J. Phys. Condens. Matter 26, 474202 (2014).

${ }^{14}$ V. A. Sydoruk, D. Xiang, S. A. Vitusevich, M. V. Petrychuk, A. Vladyka, Y. Zhang, A. Offenhäusser, V. A. Kochelap, A. E. Belyaev, and D. Mayer, J. Appl. Phys. 112, 014908 (2012).

${ }^{15}$ D. Secker and H. B. Weber, Phys. Status Solidi 244, 4176 (2007).

${ }^{16}$ L. D. Landau and E. M. Lifshits, Statistical Physics, 3rd ed. (Pergamon, 1980), pp. 25-74.

${ }^{17}$ W. Y. Wang, T. Lee, and M. A. Reed, Rep. Prog. Phys. 68, 523 (2005).

${ }^{18}$ C. H. Ko, M. J. Huang, M. D. Fu, and C. Chen, J. Am. Chem. Soc. 132, 756 (2010).

${ }^{19}$ P. Reddy, S. Y. Jang, R. A. Segalman, and A. Majumdar, Science 315, 1568 (2007).

${ }^{20}$ M. Kiguchi, S. Miura, T. Takahashi, K. Hara, M. Sawamura, and K. J. Murakoshi, Phys. Chem. C. 112, 13349 (2008).

${ }^{21}$ F. Cecchet, M. Marcaccio, M. Margotti, F. Paolucci, S. Rapino, and P. Rudolf, J. Phys. Chem. B. 110, 2241 (2006). 